\title{
SINGULAR EQUIVALENCES INDUCED BY HOMOLOGICAL EPIMORPHISMS
}

\author{
XIAO-WU CHEN
}

(Communicated by Birge Huisgen-Zimmermann)

\begin{abstract}
We prove that a certain homological epimorphism between two algebras induces a triangle equivalence between their singularity categories. Applying the result to a construction of matrix algebras, we describe the singularity categories of some non-Gorenstein algebras.
\end{abstract}

\section{INTRODUCTION}

Let $A$ be a finite dimensional algebra over a field $k$. Denote by $A$-mod the category of finitely generated left $A$-modules and by $\mathbf{D}^{b}(A$-mod $)$ the bounded derived category. Following [20, the singularity category $\mathbf{D}_{\mathrm{sg}}(A)$ of $A$ is the Verdier quotient triangulated category of $\mathbf{D}^{b}(A$-mod) with respect to the full subcategory formed by perfect complexes; see also [4, 6, 8, 14, 16, 17, 23.

The singularity category measures the homological singularity of an algebra: the algebra $A$ has finite global dimension if and only if its singularity category $\mathbf{D}_{\mathrm{sg}}(A)$ is trivial. Meanwhile, the singularity category captures the stable homological features of an algebra ([ $\underline{6})$.

A fundamental result of Buchweitz and Happel states that for a Gorenstein algebra $A$, the singularity category $\mathbf{D}_{\mathrm{sg}}(A)$ is triangle equivalent to the stable category of (maximal) Cohen-Macaulay $A$-modules ([6,14]), where the latter category is related to Tate cohomology theory $([2,6])$. This result specializes Rickard's result ([23]) on self-injective algebras. For non-Gorenstein algebras, not much is known about their singularity categories $([7,9])$.

The following concepts might be useful in the study of singularity categories. Two algebras $A$ and $B$ are said to be singularly equivalent provided that there is a triangle equivalence between $\mathbf{D}_{\mathrm{sg}}(A)$ and $\mathbf{D}_{\mathrm{sg}}(B)$. Such an equivalence is called a singular equivalence; compare [21. In this case, if $A$ is non-Gorenstein and $B$ is Gorenstein, then Buchweitz-Happel's theorem applies to give a description of $\mathbf{D}_{\mathrm{sg}}(A)$ in terms of (maximal) Cohen-Macaulay $B$-modules. We observe that a derived equivalence of two algebras, that is, a triangle equivalence between their bounded derived categories, naturally induces a singular equivalence. The converse is not true in general.

Received by the editors August 12, 2011 and, in revised form, September 6, 2012.

2010 Mathematics Subject Classification. Primary 18E30, 13E10, 16 E50.

The author was supported by the Fundamental Research Funds for the Central Universities (WK0010000024) and the National Natural Science Foundation of China (No. 11201446). 
Let $A$ be an algebra and let $J \subseteq A$ be a two-sided ideal. Following [22, we call $J$ a homological ideal provided that the canonical map $A \rightarrow A / J$ is a homological epimorphism ([12]), meaning that the naturally induced functor $\mathbf{D}^{b}(A / J$-mod) $\rightarrow$ $\mathbf{D}^{b}(A$-mod $)$ is fully faithful.

The main observation we make is as follows.

Theorem. Let $A$ be a finite dimensional $k$-algebra and let $J \subseteq A$ be a homological ideal which has finite projective dimension as an $A$-A-bimodule. Then there is a singular equivalence between $A$ and $A / J$.

This paper is structured as follows. In Section 2, we recall some ingredients and then prove the Theorem. In Section 3, we apply the Theorem to a construction of matrix algebras and then describe the singularity categories of some non-Gorenstein algebras. In particular, we give two examples which extend in different manners an example considered by Happel in [14].

\section{Proof of the Theorem}

We will present the proof of the Theorem in this section. Before that, we recall from 25] and [15] some results on triangulated categories and derived categories.

Let $\mathcal{T}$ be a triangulated category. We will denote its translation functor by [1]. For a triangulated subcategory $\mathcal{N}$, we denote by $\mathcal{T} / \mathcal{N}$ the Verdier quotient triangulated category. The quotient functor $q: \mathcal{T} \rightarrow \mathcal{T} / \mathcal{N}$ has the property that $q(X) \simeq 0$ if and only if $X$ is a direct summand of an object in $\mathcal{N}$. In particular, if $\mathcal{N}$ is a thick subcategory, that is, it is closed under direct summands, we have that $\operatorname{Ker} q=\mathcal{N}$. Here, for a triangle functor $F$, Ker $F$ denotes its essential kernel, that is, the (thick) triangulated subcategory consisting of objects on which $F$ vanishes.

The following result is well known.

Lemma 2.1. Let $F: \mathcal{T} \rightarrow \mathcal{T}^{\prime}$ be a triangle functor which allows a fully faithful right adjoint $G$. Then $F$ induces uniquely a triangle equivalence $\mathcal{T} / \operatorname{Ker} F \simeq \mathcal{T}^{\prime}$.

Proof. The existence of the induced functor follows from the universal property of the quotient functor. The result is a triangulated version of [11, Proposition I. 1.3]. For details, see [5, Propositions 1.5 and 1.6].

Let $F: \mathcal{T} \rightarrow \mathcal{T}^{\prime}$ be a triangle functor. Assume that $\mathcal{N} \subseteq \mathcal{T}$ and $\mathcal{N}^{\prime} \subseteq \mathcal{T}^{\prime}$ are triangulated subcategories satisfying $F \mathcal{N} \subseteq \mathcal{N}^{\prime}$. Then there is a uniquely induced triangle functor $\bar{F}: \mathcal{T} / \mathcal{N} \rightarrow \mathcal{T}^{\prime} / \mathcal{N}^{\prime}$.

Lemma 2.2 ([20, Lemma 1.2]). Let $F: \mathcal{T} \rightarrow \mathcal{T}^{\prime}$ be a triangle functor which has a right adjoint $G$. Assume that $\mathcal{N} \subseteq \mathcal{T}$ and $\mathcal{N}^{\prime} \subseteq \mathcal{T}^{\prime}$ are triangulated subcategories satisfying the fact that $F \mathcal{N} \subseteq \mathcal{N}^{\prime}$ and $G \mathcal{N}^{\prime} \subseteq \mathcal{N}$. Then the induced functor $\bar{F}: \mathcal{T} / \mathcal{N} \rightarrow \mathcal{T}^{\prime} / \mathcal{N}^{\prime}$ has a right adjoint $\bar{G}$. Moreover, if $G$ is fully faithful, so is $\bar{G}$.

Proof. The unit and counit of $(F, G)$ induce uniquely two natural transformations $\operatorname{Id}_{\mathcal{T} / \mathcal{N}} \rightarrow \bar{G} \bar{F}$ and $\bar{F} \bar{G} \rightarrow \operatorname{Id}_{\mathcal{T}^{\prime} / \mathcal{N}^{\prime}}$, which are the corresponding unit and counit of the adjoint pair $(\bar{F}, \bar{G})$; consult [19, Chapter IV, Section 1, Theorem 2(v)]. Note that the fully-faithfulness of $G$ is equivalent to the fact that the counit of $(F, G)$ is an isomorphism. It follows that the counit of $(\bar{F}, \bar{G})$ is also an isomorphism, which is equivalent to the fully-faithfulness of $\bar{G}$; consult [19, Chapter IV, Section 3, Theorem 1]. 
Let $k$ be a field and let $A$ be a finite dimensional $k$-algebra. Recall that $A$-mod is the category of finite dimensional left $A$-modules. We write ${ }_{A} A$ for the regular left $A$-module. Denote by $\mathbf{D}(A$-mod $)\left(r e s p . \mathbf{D}^{b}(A\right.$-mod $\left.)\right)$ the (resp. bounded) derived category of $A$-mod. We identify $A$-mod as the full subcategory of $\mathbf{D}^{b}(A$-mod $)$ consisting of stalk complexes concentrated at degree zero; see [15, Proposition I. $4.3]$.

A complex of $A$-modules is usually denoted by $X^{\bullet}=\left(X^{n}, d^{n}\right)_{n \in \mathbb{Z}}$, where $X^{n}$ are $A$-modules and the differentials $d^{n}: X^{n} \rightarrow X^{n+1}$ are homomorphisms of modules satisfying $d^{n+1} \circ d^{n}=0$. Recall that a complex in $\mathbf{D}^{b}(A$-mod) is perfect provided that it is isomorphic to a bounded complex consisting of projective modules. The full subcategory consisting of perfect complexes is denoted by $\operatorname{perf}(A)$. Recall from [6. Lemma 1.2.1] that a complex $X^{\bullet}$ in $\mathbf{D}^{b}(A$-mod) is perfect if and only if there is a natural number $n_{0}$ such that for each $A$-module $M, \operatorname{Hom}_{\mathbf{D}^{b}(A \text {-mod })}\left(X^{\bullet}, M[n]\right)=0$ for all $n \geq n_{0}$. It follows that $\operatorname{perf}(A)$ is a thick subcategory of $\mathbf{D}^{b}(A$-mod). Indeed, it is the smallest thick subcategory of $\mathbf{D}^{b}\left(A\right.$-mod) containing ${ }_{A} A$.

Let $\pi: A \rightarrow B$ be a homomorphism of algebras. The functor of restricting of scalars $\pi^{*}: B$-mod $\rightarrow A$-mod is exact, and it extends to a triangle functor $\mathbf{D}^{b}(B$-mod $) \rightarrow \mathbf{D}^{b}(A$-mod $)$, which will still be denoted by $\pi^{*}$. Following [12, we call the homomorphism $\pi$ a homological epimorphism provided that $\pi^{*}: \mathbf{D}^{b}(B$-mod $) \rightarrow$ $\mathbf{D}^{b}(A$-mod $)$ is fully faithful. By [12, Theorem 4.1(1)] this is equivalent to the fact that $\pi \otimes_{A}^{\mathbf{L}} B: B \simeq A \otimes_{A}^{\mathbf{L}} B \rightarrow B \otimes_{A}^{\mathbf{L}} B$ is an isomorphism in $\mathbf{D}\left(A^{e}\right.$-mod $)$. Here, $A^{e}=A \otimes_{k} A^{\text {op }}$ is the enveloping algebra of $A$, and we identify $A^{e}$-mod as the category of $A$ - $A$-bimodules.

Lemma 2.3 ([22, Proposition 2.2(a)]). Let $J \subseteq A$ be an ideal and let $\pi: A \rightarrow A / J$ be the canonical projection. Then $\pi$ is a homological epimorphism if and only if $J^{2}=J$ and $\operatorname{Tor}_{i}^{A}(J, A / J)=0$ for all $i \geq 1$.

In the situation of the lemma, the ideal $J$ is called a homological ideal in 22 . As a special case, we call an ideal $J$ a hereditary ideal provided that $J^{2}=J$ and $J$ is a projective $A$ - $A$-bimodule; compare [22, Lemma 3.4].

Proof. The natural exact sequence $0 \rightarrow J \rightarrow A \stackrel{\pi}{\rightarrow} A / J \rightarrow 0$ of $A$ - $A$-bimodules induces a triangle $J \rightarrow A \stackrel{\pi}{\rightarrow} A / J \rightarrow J[1]$ in $\mathbf{D}^{b}\left(A^{e}\right.$-mod). Applying the functor $-\otimes_{A}^{\mathbf{L}} A / J$, we get a triangle $J \otimes_{A}^{\mathbf{L}} A / J \rightarrow A / J \rightarrow A / J \otimes_{A}^{\mathbf{L}} A / J \rightarrow J \otimes_{A}^{\mathbf{L}} A / J[1]$. Then $\pi$ is a homological epimorphism or, equivalently, $\pi \otimes_{A}^{\mathbf{L}} A / J$ is an isomorphism if and only if $J \otimes_{A}^{\mathbf{L}} A / J=0$; see [13, Lemma I.1.7]. This is equivalent to the fact that $\operatorname{Tor}_{i}^{A}(J, A / J)=0$ for all $i \geq 0$. We note that $\operatorname{Tor}_{0}^{A}(J, A / J) \simeq J \otimes_{A} A / J \simeq J / J^{2}$.

Now we are in the position to prove the Theorem. Recall that for an algebra $A$, its singularity category $\mathbf{D}_{\mathrm{sg}}(A)=\mathbf{D}^{b}(A$-mod $) / \operatorname{perf}(A)$. Moreover, a complex $X^{\bullet}$ becomes zero in $\mathbf{D}_{\text {sg }}(A)$ if and only if it is perfect. Here, we use the fact that $\operatorname{perf}(A) \subseteq \mathbf{D}^{b}(A$-mod $)$ is a thick subcategory.

Proof of the Theorem. Write $B=A / J$. Since $J$, as an $A$ - $A$-bimodule, has finite projective dimension, so it has finite projective dimension both as a left and right $A$ module. Consider the natural exact sequence $0 \rightarrow J \rightarrow A \rightarrow B \rightarrow 0$. It follows that $B$, both as a left and right $A$-module, has finite projective dimension. Moreover, for a complex $X^{\bullet}$ in $\mathbf{D}^{b}\left(A\right.$-mod), $J \otimes_{A}^{\mathbf{L}} X^{\bullet}$ is perfect. Indeed, take a bounded projective resolution $P^{\bullet} \rightarrow J$ as an $A^{e}$-module. Then $J \otimes_{A}^{\mathbf{L}} X^{\bullet} \simeq P^{\bullet} \otimes_{A} X^{\bullet}$. This is a perfect complex, since each left $A$-module $P^{i} \otimes_{A} X^{j}$ is projective. 
Denote by $\pi: A \rightarrow B$ the canonical projection. By the assumption, the functor $\pi^{*}: \mathbf{D}^{b}(B$-mod $) \rightarrow \mathbf{D}^{b}(A$-mod $)$ is fully faithful. Since $\pi^{*}(B)={ }_{A} B$ is perfect, the functor $\pi^{*}$ sends perfect complexes to perfect complexes. Then it induces a triangle functor $\overline{\pi^{*}}: \mathbf{D}_{\mathrm{sg}}(B) \rightarrow \mathbf{D}_{\mathrm{sg}}(A)$. We will show that $\overline{\pi^{*}}$ is an equivalence.

The functor $\pi^{*}: \mathbf{D}^{b}(B$-mod $) \rightarrow \mathbf{D}^{b}(A$-mod $)$ has a left adjoint $F=B \otimes_{A}^{\mathbf{L}}$ $-: \mathbf{D}^{b}(A$-mod $) \rightarrow \mathbf{D}^{b}(B$-mod $)$. Here we use the fact that the right $A$-module $B_{A}$ has finite projective dimension. Since $F$ sends perfect complexes to perfect complexes, we have the induced triangle functor $\bar{F}: \mathbf{D}_{\mathrm{sg}}(A) \rightarrow \mathbf{D}_{\mathrm{sg}}(B)$. By Lemma 2.2 we have the adjoint pair $\left(\bar{F}, \bar{\pi}^{*}\right)$; moreover, the functor $\bar{\pi}^{*}$ is fully faithful. By Lemma 2.1 there is a triangle equivalence $\mathbf{D}_{\mathrm{sg}}(A) / \operatorname{Ker} \bar{F} \simeq \mathbf{D}_{\mathrm{sg}}(B)$.

It remains to show that the essential kernel Ker $\bar{F}$ is trivial. For this, we assume that a complex $X^{\bullet}$ lies in Ker $\bar{F}$. This means that the complex $F\left(X^{\bullet}\right)$ in $\mathbf{D}^{b}\left(B\right.$-mod) is perfect. Since $\pi^{*}$ preserves perfect complexes, it follows that $\pi^{*} F\left(X^{\bullet}\right)$ is also perfect. The natural exact sequence $0 \rightarrow J \rightarrow A \rightarrow B \rightarrow 0$ induces a triangle $J \otimes_{A}^{\mathbf{L}} X^{\bullet} \rightarrow X^{\bullet} \rightarrow \pi^{*} F\left(X^{\bullet}\right) \rightarrow J \otimes_{A}^{\mathbf{L}} X^{\bullet}[1]$ in $\mathbf{D}^{b}(A$-mod). Recall that $J \otimes_{A}^{\mathbf{L}} X^{\bullet}$ is perfect. It follows that $X^{\bullet}$ is perfect, since perf $(A) \subseteq \mathbf{D}^{b}(A$-mod) is a triangulated subcategory. The proves that $X^{\bullet}$ is zero in $\mathbf{D}_{\mathrm{sg}}(A)$.

The following special case of the Theorem is of interest.

Corollary 2.4. Let $A$ be a finite dimensional algebra and $J \subseteq A$ a hereditary ideal. Then we have a triangle equivalence $\mathbf{D}_{\mathrm{sg}}(A) \simeq \mathbf{D}_{\mathrm{sg}}(A / J)$.

Proof. It suffices to observe by Lemma 2.3 that $J$ is a homological ideal.

\section{EXAmples}

In this section, we will describe a construction of matrix algebras to illustrate Corollary 2.4. In particular, the singularity categories of some non-Gorenstein algebras are described.

The following construction is similar to [18, Section 4]. Let $A$ be a finite dimensional algebra over a field $k$. Let ${ }_{A} M$ and $N_{A}$ be a left and right $A$-module, respectively. Then $M \otimes_{k} N$ becomes an $A$ - $A$-bimodule. Consider an $A$ - $A$-bimodule monomorphism $\phi: M \otimes_{k} N \rightarrow A$. Then $\operatorname{Im} \phi$ is a two-sided ideal of $A$. We require further that $(\operatorname{Im} \phi) M=0$ and $N(\operatorname{Im} \phi)=0$. The matrix $\Gamma=\left(\begin{array}{cc}A & M \\ N & k\end{array}\right)$ becomes an associative algebra via the following multiplication:

$$
\left(\begin{array}{cc}
a & m \\
n & \lambda
\end{array}\right)\left(\begin{array}{cc}
a^{\prime} & m^{\prime} \\
n^{\prime} & \lambda^{\prime}
\end{array}\right)=\left(\begin{array}{cc}
a a^{\prime}+\phi\left(m \otimes n^{\prime}\right) & a m^{\prime}+\lambda^{\prime} m \\
n a^{\prime}+\lambda n^{\prime} & \lambda \lambda^{\prime}
\end{array}\right) .
$$

For the associativity, we need the above requirement on $\operatorname{Im} \phi$.

Proposition 3.1. Keep the notation and assumption as above. Then there is a triangle equivalence $\mathbf{D}_{\mathrm{sg}}(\Gamma) \simeq \mathbf{D}_{\mathrm{sg}}(A / \operatorname{Im} \phi)$.

Proof. Set $J=\Gamma e \Gamma$ with $e=\left(\begin{array}{ll}0 & 0 \\ 0 & 1\end{array}\right)$. Observe that $\Gamma / J=A / \operatorname{Im} \phi$. The ideal $J$ is hereditary: $J^{2}=J$ is clear, while the natural map $\Gamma e \otimes_{k} e \Gamma \rightarrow J$ is an isomorphism of $\Gamma$ - $\Gamma$-bimodules and then $J$ is a projective $\Gamma$ - $\Gamma$-bimodule. The isomorphism uses the assumption that $\phi$ is mono. Then we apply Corollary 2.4. 
Remark 3.2. The above construction contains the one-point extension and coextension of algebras, where $M$ or $N$ is zero. Hence Proposition 3.1 contains the results in [9, Section 4].

We will illustrate Proposition 3.1 by three examples. Two of these examples extend an example considered by Happel in [14. In particular, based on results in [9], we obtain descriptions of the singularity categories of some non-Gorenstein algebras.

Recall from 14 that an algebra $A$ is Gorenstein provided that both as a left and right module, the regular module $A$ has finite injective dimension. It follows from [6. Theorem 4.4.1] and [14, Theorem 4.6] that in the Gorenstein case, the singularity category $\mathbf{D}_{\mathrm{sg}}(A)$ is Hom-finite. This means that all Hom spaces in $\mathbf{D}_{\mathrm{sg}}(A)$ are finite dimensional over $k$.

For algebras given by quivers and relations, we refer to [1, Chapter III].

Example 3.3. Let $\Gamma$ be the $k$-algebra given by the following quiver $Q$ with relations $\left\{x^{2}, \delta x, \beta x, x \gamma, x \alpha, \beta \gamma, \delta \alpha, \beta \alpha, \delta \gamma, \alpha \beta-\gamma \delta\right\}$. We write the concatenation of paths from right to left.

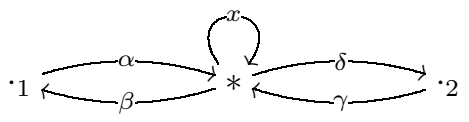

We have in $\Gamma$ that $1=e_{1}+e_{*}+e_{2}$, where the $e$ 's are the primitive idempotents corresponding to the vertices. Set $\Gamma^{\prime}=\Gamma / \Gamma e_{1} \Gamma$. It is an algebra with radical square zero, whose quiver is obtained from $Q$ by removing the vertex 1 and the adjacent arrows.

We identify $\Gamma$ with $\left(\begin{array}{cc}A & k \alpha \\ k \beta & k\end{array}\right)$, where the $k$ in the southeast corner is identified with $e_{1} \Gamma e_{1}$, and $A=\left(1-e_{1}\right) \Gamma\left(1-e_{1}\right)$. The corresponding $\operatorname{Im} \phi$ equals $k \alpha \beta$, and we have $A / \operatorname{Im} \phi=\Gamma^{\prime}$; consult the proof of Proposition 3.1. Then Proposition 3.1 yields a triangle equivalence $\mathbf{D}_{\mathrm{sg}}(\Gamma) \simeq \mathbf{D}_{\mathrm{sg}}\left(\Gamma^{\prime}\right)$.

The triangulated category $\mathbf{D}_{\mathrm{sg}}\left(\Gamma^{\prime}\right)$ is completely described in [9] (see also 24]); in particular, it is not Hom-finite. More precisely, it is equivalent to the category of finitely generated projective modules on a von Neumann regular algebra. The algebra $\Gamma^{\prime}$, or rather its Koszul dual, is related to the noncommutative space of Penrose tilings via the work of Smith; see [24, Theorem 7.2 and Example]. We point out that the algebra $\Gamma$ is non-Gorenstein, since $\mathbf{D}_{\mathrm{sg}}(\Gamma)$ is not Hom-finite.

Example 3.4. Let $\Gamma$ be the $k$-algebra given by the following quiver $Q$ with relations $\left\{x_{1}^{2}, x_{2}^{2}, x_{1} \alpha_{1}, x_{2} \alpha_{1}, \beta_{2} \alpha_{1}, \beta_{2} \alpha_{1}, x_{1} \alpha_{2}, x_{2} \alpha_{2}, \beta_{1} \alpha_{2}, \beta_{2} \alpha_{2}, \alpha_{1} \beta_{1}-x_{1} x_{2}, \alpha_{2} \beta_{2}-x_{2} x_{1}\right\}$ :

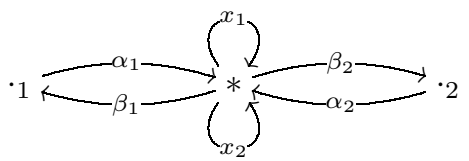

We claim that there is a triangle equivalence $\mathbf{D}_{\mathrm{sg}}(\Gamma) \simeq \mathbf{D}_{\mathrm{sg}}\left(k\left\langle x_{1}, x_{2}\right\rangle /\left(x_{1}, x_{2}\right)^{2}\right)$. Here, $k\left\langle x_{1}, x_{2}\right\rangle$ is the free algebra with two variables.

We point out that the triangulated category $\mathbf{D}_{\mathrm{sg}}\left(k\left\langle x_{1}, x_{2}\right\rangle /\left(x_{1}, x_{2}\right)^{2}\right)$ is described completely in [9, Example 3.11], where related results are contained in 3, Section 10]. Similar to the example above, this algebra $\Gamma$ is non-Gorenstein. 
To see the claim, we observe that the quiver $Q$ has two loops and two 2-cycles. The proof is done by "removing the 2-cycles". We have a natural isomorphism $\Gamma=\left(\begin{array}{cc}A & k \alpha_{1} \\ k \beta_{1} & k\end{array}\right)$, where $k=e_{1} \Gamma e_{1}$ and $A=\left(1-e_{1}\right) \Gamma\left(1-e_{1}\right)$. We observe that Proposition 3.1 applies with the corresponding $\operatorname{Im} \phi=k \alpha_{1} \beta_{1}$. Set $A / \operatorname{Im} \phi=\Gamma^{\prime}$. So $\mathbf{D}_{\mathrm{sg}}(\Gamma) \simeq \mathbf{D}_{\mathrm{sg}}\left(\Gamma^{\prime}\right)$. The quiver of $\Gamma^{\prime}$ is obtained from $Q$ by removing the vertex 1 and the adjacent arrows, while its relations are obtained from the ones of $\Gamma$ by replacing $\alpha_{1} \beta_{1}-x_{1} x_{2}$ with $x_{1} x_{2}$. Similarly, $\Gamma^{\prime}=\left(\begin{array}{cc}A^{\prime} & k \alpha_{2} \\ k \beta_{2} & k\end{array}\right)$ with $k=e_{2} \Gamma^{\prime} e_{2}$ and $A^{\prime}=e_{*} \Gamma^{\prime} e_{*}$. Then Proposition 3.1 applies again, and we get the equivalence $\mathbf{D}_{\mathrm{sg}}\left(\Gamma^{\prime}\right) \simeq \mathbf{D}_{\mathrm{sg}}\left(k\left\langle x_{1}, x_{2}\right\rangle /\left(x_{1}, x_{2}\right)^{2}\right)$.

This example generalizes directly to a quiver with $n$ loops and $n 2$-cycles with similar relations. The corresponding statement for the case $n=1$ is implicitly contained in [14, 2.3 and 4.8].

The last example is a Gorenstein algebra.

Example 3.5. Let $r \geq 2$. Consider the following quiver $Q$ consisting of three 2-cycles and a central 3-cycle $Z_{3}$. We identify $\gamma_{3}$ with $\gamma_{0}$ and denote by $p_{i}$ the path in the central cycle starting at vertex $i$ of length 3 .

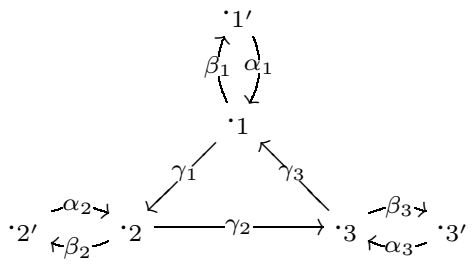

Let $\Gamma$ be the $k$-algebra given by the quiver $Q$ with relations $\left\{\beta_{i} \alpha_{i}, \gamma_{i} \alpha_{i}, \beta_{i} \gamma_{i-1}, \alpha_{i} \beta_{i}-\right.$ $\left.p_{i}^{r} \mid i=1,2,3\right\}$. We point out that in $\Gamma$ all paths in the central cycle of length strictly larger than $3 r+1$ vanish.

Set $A=k Z_{3} /\left(\gamma_{1}, \gamma_{2}, \gamma_{3}\right)^{3 r}$, where $k Z_{3}$ is the path algebra of the central 3cycle $Z_{3}$. The algebra $A$ is self-injective and Nakayama ([1, p.111]). Denote by $A$-mod the stable category of $A$-modules; it is naturally a triangulated category (see [13, Theorem I.2.6]).

We claim that there is a triangle equivalence $\mathbf{D}_{\mathrm{sg}}(\Gamma) \simeq A$-mod.

For the claim, we observe an isomorphism $A=\Gamma / \Gamma\left(e_{1^{\prime}}+e_{2^{\prime}}+e_{3^{\prime}}\right) \Gamma$. We argue as in Example 3.4 by removing the three 2-cycles and applying Proposition 3.1 repeatedly. Then we get a triangle equivalence $\mathbf{D}_{\mathrm{sg}}(\Gamma) \simeq \mathbf{D}_{\mathrm{sg}}(A)$. Finally, by [23. Theorem 2.1] we have a triangle equivalence $\mathbf{D}_{\mathrm{sg}}(A) \simeq A$-mod. Then we are done.

We point out that the algebra $\Gamma$ is Gorenstein with self-injective dimension two. Hence by [6, Theorem 4.4.1] and [14, Theorem 4.6] there is a triangle equivalence $\mathbf{D}_{\mathrm{sg}}(\Gamma) \simeq \underline{\operatorname{MCM}}(\Gamma)$, where $\underline{\operatorname{MCM}}(\Gamma)$ denotes the stable category of (maximal) Cohen-Macaulay $\Gamma$-modules. Then we have a triangle equivalence

$$
\underline{\operatorname{MCM}}(\Gamma) \simeq A \text { - } \underline{\bmod } .
$$

We mention that $\Gamma$ is a special biserial algebra of finite representation type (by [10, Lemma II.8.1]). It would be interesting to identify (maximal) Cohen-Macaulay $\Gamma$-modules in the Auslander-Reiten quiver of $\Gamma$. 
This example generalizes directly to a quiver with $n$ 2-cycles and a central $n$ cycle with similar relations. The case where $n=1$ and $r=2$ coincides with the examples considered in [14, 2.3 and 4.8].

\section{ACKNOWLEDGEMENTS}

The author thanks the referee for useful comments and Huanhuan Li for helpful discussions. The results of this paper partially answer a question that was raised by Professor Changchang Xi during a conference held in Jinan in June 2011.

\section{REFERENCES}

[1] Maurice Auslander, Idun Reiten, and Sverre O. Smalø, Representation theory of Artin algebras, Cambridge Studies in Advanced Mathematics, vol. 36, Cambridge University Press, Cambridge, 1995. MR1314422 (96c:16015)

[2] Luchezar L. Avramov and Alex Martsinkovsky, Absolute, relative, and Tate cohomology of modules of finite Gorenstein dimension, Proc. London Math. Soc. (3) 85 (2002), no. 2, 393440, DOI 10.1112/S0024611502013527. MR1912056 (2003g:16009)

[3] Luchezar L. Avramov and Oana Veliche, Stable cohomology over local rings, Adv. Math. 213 (2007), no. 1, 93-139, DOI 10.1016/j.aim.2006.11.012. MR2331239 (2008f:13020)

[4] Apostolos Beligiannis, The homological theory of contravariantly finite subcategories: Auslander-Buchweitz contexts, Gorenstein categories and (co-)stabilization, Comm. Algebra 28 (2000), no. 10, 4547-4596, DOI 10.1080/00927870008827105. MR1780017 (2001g:18012)

[5] A. I. Bondal and M. M. Kapranov, Representable functors, Serre functors, and reconstructions (Russian), Izv. Akad. Nauk SSSR Ser. Mat. 53 (1989), no. 6, 1183-1205, 1337; English transl., Math. USSR-Izv. 35 (1990), no. 3, 519-541. MR1039961 (91b:14013)

[6] R. O. Buchweitz, Maximal Cohen-Macaulay Modules and Tate Cohomology over Gorenstein Rings, unpublished manuscript, 1987.

[7] Xiao-Wu Chen, Singularity categories, Schur functors and triangular matrix rings, Algebr. Represent. Theory 12 (2009), no. 2-5, 181-191, DOI 10.1007/s10468-009-9149-2. MR.2501179 (2010c:18015)

[8] Xiao-Wu Chen, Relative singularity categories and Gorenstein-projective modules, Math. Nachr. 284 (2011), no. 2-3, 199-212, DOI 10.1002/mana.200810017. MR2790881 (2012f:18024)

[9] Xiao-Wu Chen, The singularity category of an algebra with radical square zero, Doc. Math. 16 (2011), 921-936. MR2880676

[10] Karin Erdmann, Blocks of tame representation type and related algebras, Lecture Notes in Mathematics, vol. 1428, Springer-Verlag, Berlin, 1990. MR.1064107 (91c:20016)

[11] P. Gabriel and M. Zisman, Calculus of fractions and homotopy theory, Ergebnisse der Mathematik und ihrer Grenzgebiete, Band 35, Springer-Verlag New York, Inc., New York, 1967. MR0210125 (35 \#1019)

[12] Werner Geigle and Helmut Lenzing, Perpendicular categories with applications to representations and sheaves, J. Algebra 144 (1991), no. 2, 273-343, DOI 10.1016/0021-8693(91)90107-J. MR:1140607 (93b:16011)

[13] Dieter Happel, Triangulated categories in the representation theory of finite-dimensional algebras, London Mathematical Society Lecture Note Series, vol. 119, Cambridge University Press, Cambridge, 1988. MR935124 (89e:16035)

[14] Dieter Happel, On Gorenstein algebras (Bielefeld, 1991), Progr. Math., vol. 95, Birkhäuser, Basel, 1991, pp. 389-404. MR1112170(92k:16022)

[15] Robin Hartshorne, Residues and duality, Lecture notes of a seminar on the work of A. Grothendieck, given at Harvard 1963/64. With an appendix by P. Deligne. Lecture Notes in Mathematics, No. 20, Springer-Verlag, Berlin, 1966. MR0222093 (36 \#5145)

[16] Bernhard Keller and Dieter Vossieck, Sous les catégories dérivées (French, with English summary), C. R. Acad. Sci. Paris Sér. I Math. 305 (1987), no. 6, 225-228. MR.907948 $(88 \mathrm{~m}: 18014)$

[17] Henning Krause, The stable derived category of a Noetherian scheme, Compos. Math. 141 (2005), no. 5, 1128-1162, DOI 10.1112/S0010437X05001375. MR2157133 (2006e:18019) 
[18] Steffen Koenig and Hiroshi Nagase, Hochschild cohomology and stratifying ideals, J. Pure Appl. Algebra 213 (2009), no. 5, 886-891, DOI 10.1016/j.jpaa.2008.10.012. MR2494378 (2009m:16020)

[19] Saunders Mac Lane, Categories for the working mathematician, 2nd ed., Graduate Texts in Mathematics, vol. 5, Springer-Verlag, New York, 1998. MR.1712872 (2001j:18001)

[20] D. O. Orlov, Triangulated categories of singularities and D-branes in Landau-Ginzburg models (Russian, with Russian summary), Tr. Mat. Inst. Steklova 246 (2004), no. Algebr. Geom. Metody, Svyazi i Prilozh., 240-262; English transl., Proc. Steklov Inst. Math. 3 (246) (2004), 227-248. MR2101296(2006i:81173)

[21] D. O. Orlov, Triangulated categories of singularities, and equivalences between LandauGinzburg models (Russian, with Russian summary), Mat. Sb. 197 (2006), no. 12, 117-132, DOI 10.1070/SM2006v197n12ABEH003824; English transl., Sb. Math. 197 (2006), no. 11-12, 1827-1840. MR2437083 (2009g:14013)

[22] José A. de la Peña and Changchang Xi, Hochschild cohomology of algebras with homological ideals, Tsukuba J. Math. 30 (2006), no. 1, 61-79. MR2248284(2007j:16014)

[23] Jeremy Rickard, Derived categories and stable equivalence, J. Pure Appl. Algebra 61 (1989), no. 3, 303-317, DOI 10.1016/0022-4049(89)90081-9. MR1027750 (91a:16004)

[24] S. Paul Smith, Category equivalences involving graded modules over path algebras of quivers, Adv. Math. 230 (2012), no. 4-6, 1780-1810, DOI 10.1016/j.aim.2012.03.031. MR2927354

[25] P. Deligne, Cohomologie étale, Lecture Notes in Mathematics, Vol. 569, Springer-Verlag, Berlin, 1977. Séminaire de Géométrie Algébrique du Bois-Marie SGA 4 $\frac{1}{2}$; avec la collaboration de J. F. Boutot, A. Grothendieck, L. Illusie et J. L. Verdier. MR0463174 (57 \#3132)

Wu Wen-Tsun Key Laboratory of Mathematics, University of Science and Technology of China, Chinese Academy of Sciences, Hefei 230026, Anhui, People's Republic of CHINA

E-mail address: xwchen@mail.ustc.edu.cn

$U R L:$ http: //home.ustc.edu.cn/ ${ }^{\text {xwchen }}$ 